\title{
Analyse des „Ubiquitin-Codes“ mithilfe Protease-resistenter Ubiquitinketten
}

\section{TATJANA SCHNEIDER, DANIEL SCHNEIDER, ANDREAS MARX, MARTIN SCHEFFNER FACHBEREICHE CHEMIE UND BIOLOGIE, UNIVERSITÄT KONSTANZ}

\section{Modification of proteins by ubiquitin plays a fundamental role in maintain- ing a functional proteome. Proteins can be modified by single ubiquitin moieties or by various types of ubiquitin chains differing in linkage type and topology. Different ubiquitin chains are assumed to signal target pro- teins for different fates, but how this is achieved is only partially under- stood. We developed an easy-to-use strategy to generate large amounts of linkage-defined, non-hydrolyzable ubiquitin chains and show their potential to dissect ubiquitin signalling.}

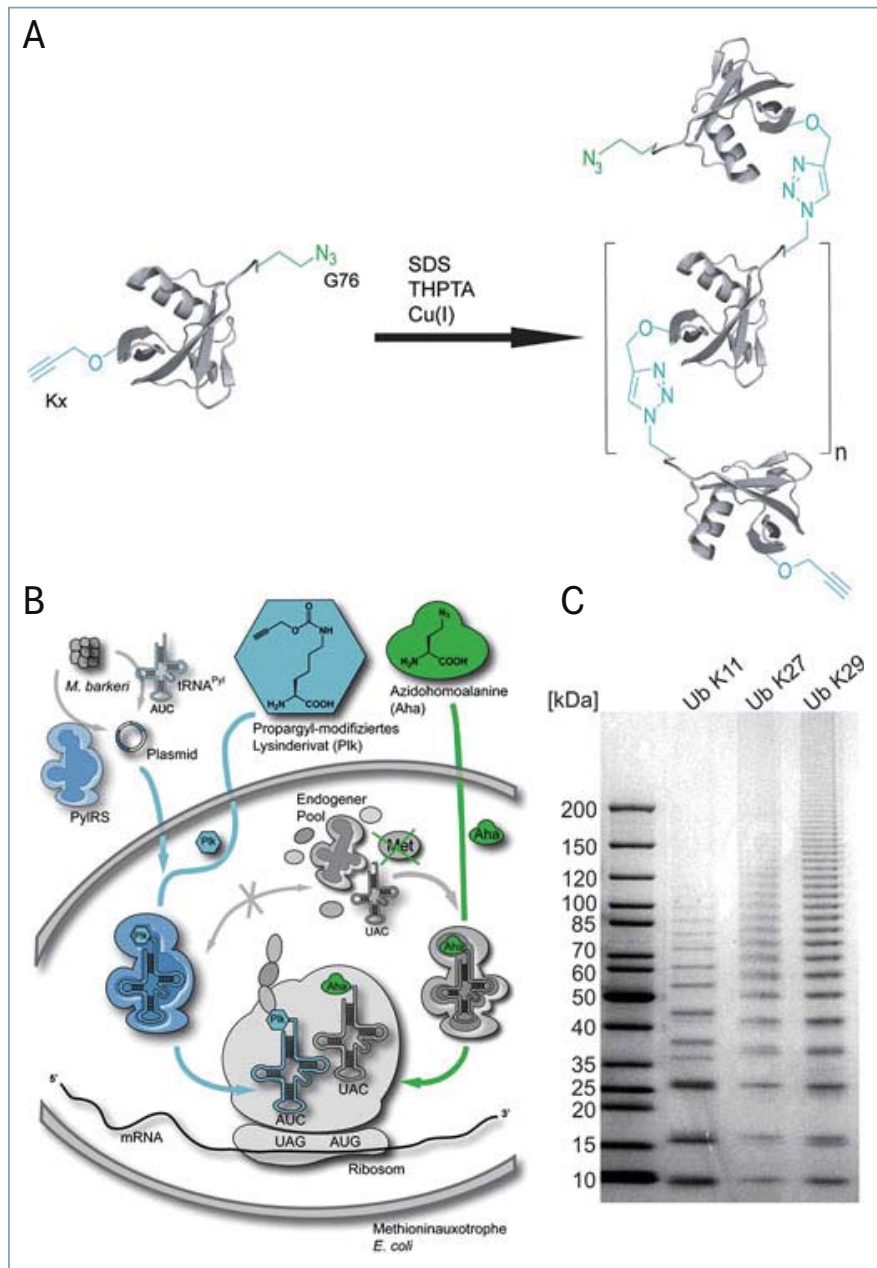
Proteine - werden
— Die Verknüpfung Substratproteinen (Ubiquitinierung) ist eine häufige und tionale Modifika in eukaryotischen Zellen. Nahezu alle intrazellulären eukaryotischen Proteine - darunter eine Viel- zumindest einmal in ihrer Lebenszeit ubiquitiniert [1-3]. Entsprechend tragen Fehlfunktionen in der Ubiquitinierungsmaschinerie zur Entstehung diverser Krankheitsbilder bei, darunter verschiedene Formen von Krebs und neurodegenerative Erkrankungen [4]. Es sind mehrere Arten der Ubiquitinierung bekannt, aber alle werden durch das Zusammenspiel von mindestens drei verschiedenen Enzymklassen katalysiert: den E1-Ubiquitin-aktivierenden Enzymen, den E2-Ubiquitin-konjugierenden Enzymen und den E3-Ubiquitin-Proteinligasen [5]. Im einfachsten Fall wird ein Ubiquitinmolekül mit dem Substrat über eine Isopeptidbindung zwischen der $\varepsilon$-Aminogruppe eines spezifischen Lysins des Substrats und der Carboxylgruppe des C-terminalen Glycins von Ubiquitin verknüpft (Mono-Ubiquitinierung). Zusätzlich kann Ubiquitin selbst als Substrat dienen, was die Bildung von Ubiquitinketten zur Folge hat (Poly-Ubiquitinierung). Ubiquitin verfügt über sieben Lysine (K6, K11, K27, K29, K33, K48 und K63), und da alle, ebenso wie die N-terminale Aminogruppe, in unterschiedlichen Kombinationen zur Kettenbildung verwendet werden können, ist Poly-Ubiquitinierung ein Oberbegriff für eine Vielzahl möglicher Ubiquitinmodifikationen [1]. Dabei scheint die jeweilige Position der Verknüpfung zwischen den Ubiquitinein-

\begin{abstract}
4 Abb. 1: Herstellung von Ubiquitinketten durch Click-Chemie. A, Ubiquitin wird durch Einbau der nicht-natürlichen Aminosäuren Plk anstelle eines bestimmten Lysinrestes (Kx) und Azidohomoalanin (Aha) anstatt des C-terminalen Glycins (G76) mit einer Alkin- und einer Azidfunktionalität ausgestattet. Die resultierenden Ubiquitinmoleküle werden durch Inkubation mit $\mathrm{Cu}(\mathrm{l})$, dem $\mathrm{Cu}(\mathrm{I})$-stabilisierenden Liganden THPTA (Tris(3-hydroxypropyltriazolylmethyl)amin) und SDS (Sodiumdodecylsulfat) mittels ClickChemie über Triazolringe verbunden und bilden Ubiquitinketten. B, Zum Einbau von Aha und Plk werden methioninauxotrophe Eschericha coli B834 (DE3) mit einem Plasmid transformiert, das die Pyrrolysyl-tRNASynthetase (PyIRS) und die tRNA ${ }^{\text {Pyl }}$ codiert. Die Expression in Methioninfreiem Medium, das Aha und PIk enthält, ermöglicht den Einbau beider Aminosäuren. C, Analyse der gebildeten Ubiquitinketten durch SDS-PAGE. Die gezeigten Ketten unterscheiden sich im Verknüpfungstyp; von links nach rechts wurden die Lysinreste K11, K27 bzw. K29 durch Plk ersetzt und dienten als Verknüpfungspunkt der einzelnen Ubiquitinmoleküle. Modifiziert nach [10].
\end{abstract}


heiten innerhalb einer Kette das Schicksal des Zielproteins zu bestimmen („Ubiquitin-Code“). Am besten charakterisiert sind homotypische K48-verknüpfte Ketten (das heißt, innerhalb der Kette dient ausschließlich K48 als Verknüpfungspunkt), die Proteine für den Abbau durch das 26SProteasom markieren [6]. Ähnlich führen K11-verknüpfte Ubiquitinketten zum proteasomalen Abbau. Im Gegensatz dazu haben K63- und N-terminal verknüpfte Ketten nicht-proteolytische Funktionen bei Prozessen wie DNA-Reparatur, Endozytose und Signaltransduktion. Die Existenz der anderen homotypischen sowie von gemischten und verzweigten Ubiquitinketten wurde zwar nachgewiesen, die Aufklärung ihrer Funktionen befindet sich aber noch in den Anfängen [1, 7].

\section{Methoden zur Herstellung von Ubiquitinketten}

Eine große Hürde in der Erforschung unterschiedlicher Ubiquitinketten ist die Erzeugung homogen verknüpfter Ubiquitinketten und vor allem von entsprechend poly-ubiquitinierten Proteinen in Mengen, die eine biochemische Charakterisierung erlauben. Eine Möglichkeit ist die enzymatische Darstellung freier Ubiquitinketten, was allerdings nur unter erheblichem Aufwand durchführbar ist. Auch zeigen die Enzyme der Ubiquitinierungskaskade in vitro eine geringe Selektivität für eine Aminosäure im Substrat, weswegen eine definierte Poly-Ubiquitinierung von Substratproteinen meist nicht realisierbar ist. Um jeden möglichen Verknüpfungstyp zu generieren, wurden zahlreiche chemische Methoden entwickelt, wobei FestphasenPeptidsynthesen oder chemische Prozessierung von Ubiquitinmolekülen nötig sind, um die für die Kopplung zweier Ubiquitinmoleküle benötigten reaktiven Gruppen zu generieren [8].

\section{Per Click-Chemie zu allen homotypischen Kettentypen}

Wir haben eine einfache Methode entwickelt, die ausschließlich auf molekularbiologischen und proteinbiochemischen Standardmethoden beruht und es erlaubt, Ubiquitinketten jedes gewünschten Verknüpfungstyps zu erzeugen [9]. Das Prinzip der Methode beruht auf der Verknüpfung entsprechend funktionalisierter Ubiquitinmoleküle mittels bioorthogonaler $\mathrm{Cu}(\mathrm{I})$-katalysierter Azid-Alkin-Cycloaddi- tion („Click-Chemie“), wodurch Triazolbindungen zwischen den Ubiquitineinheiten entstehen (Abb. 1A). Zum simultanen Einbringen der benötigten Azid- und Alkingruppen in Ubiquitin werden verschiedene Methoden zur Erweiterung des genetischen Codes miteinander kombiniert und entsprechende nicht-natürliche Aminosäuren verwendet. Mittels selective pressure incorporation wird das C-terminale Glycin durch das Azid-funktionalisierte Methionin-Analogon Azidohomoalanin (Aha) ersetzt. Gleichzeitig wird eines der sieben Lysine unter Verwendung der amber codon suppression mit dem Alkinfunktionalisierten Lysin-Analogon Plk substituiert (Abb. 1B). Auf diese Weise lassen sich durch rekombinante Genexpression in Escherichia coli und anschließende Proteinreinigung bifunktionale Ubiquitin-Bausteine herstellen, die unter $\mathrm{Cu}(\mathrm{I})$-Katalyse effizient polymerisiert werden können [9, 10]. Es entstehen lange, teilweise aus mehr als 30 Untereinheiten bestehende, homogen verknüpfte Ketten (Abb. 1C). Unter Verwendung eines verknüpfungsspezifischen Antikörpers gegen Ubiquitinketten konnten wir nachweisen, dass die Triazolverknüpfungen zwischen den Monomeren die Topologie der Ketten und somit die Erkennung durch andere Proteine nicht wesentlich beeinflussen. Des Weiteren ist die Methode nicht auf die Generierung von freien Ubiquitinketten beschränkt. Vielmehr kann sie zur Darstellung von Proteinen, die an definierten Positionen poly-ubiquitiniert sind, adaptiert werden, wie wir am Beispiel der DNAPolymerase beta gezeigt haben [10].

\section{Einfluss verschiedener Kettentypen auf die Meiose in Xenopus-Extrakten}

Um zu zeigen, dass die artifiziell verknüpften Ketten dazu geeignet sind, Ubiquitin-abhängige Prozesse in biologisch relevanten Systemen zu untersuchen, wurden Extrakte von Xenopus laevis-Oozyten verwendet - ein beliebtes Modellsystem zur Untersuchung des Zellzyklus. Die Oozyten sind bis zur Befruchtung in der Metaphase der zweiten meiotischen Teilung arretiert, wobei die Befruchtung in Oozyten-Extrakten durch Zugabe von $\mathrm{Ca}^{2+}$ simuliert werden kann. Dies führt zur Modifikation von Cyclin-B durch K11-verknüpfte Ubiquitinketten, was Cyclin-B für den proteasomalen Abbau markiert und 


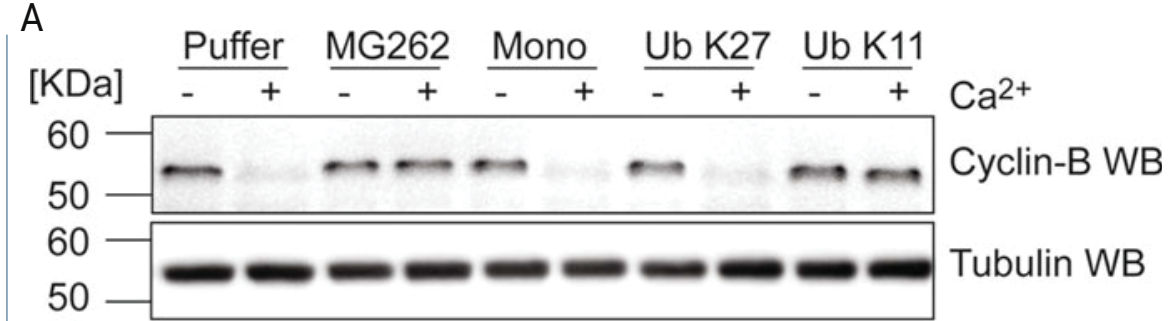

B

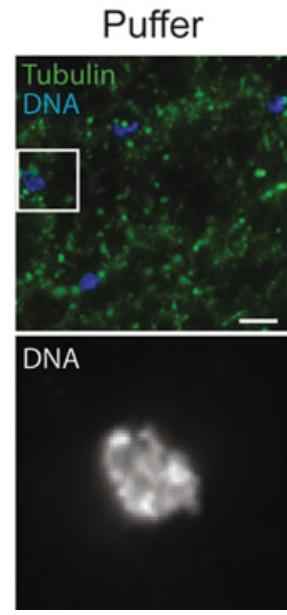

MG262

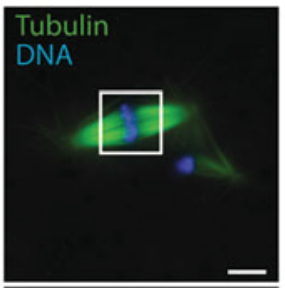

DNA

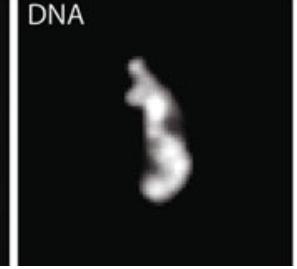

Ub K11

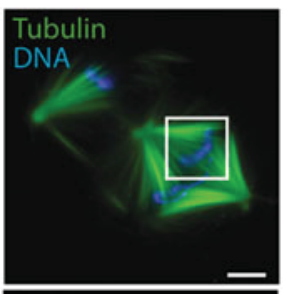

DNA

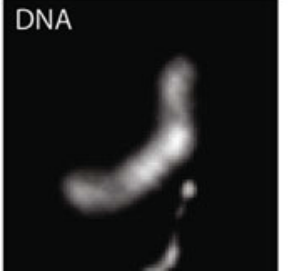

$\Delta$ Abb. 2: Artifiziell hergestellte K11-verknüpfte Ubiquitinketten inhibieren den Abbau von CyclinB im Oozytenextrakt von Xenopus laevis. A, Zugabe von $\mathrm{Ca}^{2+}$ führt zum Abbau von Cyclin-B in Extrakten, die mit monomerem Ubiquitin (Mono) oder K27-verknüpften Ketten (Ub K27) behandelt wurden, während der Abbau durch K11-verknüpfte Ketten (Ub K11) oder dem Proteasominhibitor MG262 inhibiert wird. Tubulin dient als Ladekontrolle. WB: Western Blot. B, Nach Zugabe von $\mathrm{Ca}^{2+}$ verlassen Puffer-behandelte Extrakte das meiotische Stadium. Dies führt zum Auftreten von Interphasenchromatin (Vergrößerung des markierten Bereichs in der unteren Reihe) und der Abwesenheit der Mikrotubuli-Spindelstrukturen. Extrakte, die mit MG262 oder K11-verknüpften Ketten behandelt wurden, weisen metaphasentypische bipolare Spindeln und kondensiertes Chromatin auf. Maßstabsbalken: $10 \mu \mathrm{m}$, modifiziert nach [10].

so den Austritt aus der Metaphase einleitet [11, 12]. Die Zugabe der freien K11-Ubiquitinketten zu den Xenopus-Extrakten führte zu einer effizienten Inhibition des Abbaus von Cyclin-B und zum Verbleib in der Metaphase, was sich in der Aufrechterhaltung der kondensierten Struktur des Chromatins und des intakten bipolaren Spindelapparat widerspiegelt (Abb. 2). Dagegen wurde der Abbau von Cyclin-B durch Zugabe der artifiziell verknüpften K27- oder K29-Ketten nicht verhindert, sodass nach $\mathrm{Ca}^{2+}$-Zugabe der Austritt aus der Meiose eingeleitet wurde [10]. Diese Daten zeigen eindrücklich, dass die artifiziell erzeugten Ubiquitinketten, die gegenüber ubiquitär vorkommenden Deubiquitinasen (DUBs) aufgrund ihrer artifiziellen Verknüpfung resistent sind, in Zellextrakten funktionell sind und sich somit als Werkzeuge zur Entschlüsselung des Ubiquitin-Codes eignen.

\section{Ausblick}

Wir beschreiben eine einfache Methode zur Herstellung homotypisch verknüpfter Ubiquitinketten sowie poly-ubiquitinierter Pro- teine durch eine Kombination von Click-Chemie und der Erweiterung des genetischen Codes. Die artifiziell verknüpften Ketten sind in Zellextrakten funktionell und zeichnen sich durch Resistenz gegenüber der hydrolytischen Aktivität von DUBs aus. Sie ermöglichen somit neue Anwendungen in Zelllysaten, wie beispielsweise die Isolation verknüpfungsspezifischer Interaktionspartner mittels KoPräzipitations-Experimenten, und tragen so zur Aufklärung der biochemischen und zellulären Funktionen unterschiedlicher Ubiquitinketten bei.

\section{Danksagung}

Wir danken der Deutschen Forschungsgemeinschaft im Rahmen des SFB 969 Chemical and Biological Principles of Cellular Proteostasis, der Carl-Zeiss-Stiftung (Stipendium für T. Schneider) und der Konstanz Research School Chemical Biology für finanzielle Unterstützung. Wir bedanken uns bei Daniel Rösner und Saurav Malhotra für die Zusammenarbeit am Projekt und Erstellen von Abbildungen.

\section{Literatur}

[1] Komander D, Rape M (2012) The ubiquitin code. Annu Rev Biochem 81:203-229

[2] Hershko A, Ciechanover A (1998) The ubiquitin system. Annu Rev Biochem 67:425-479

[3] Spasser L, Brik A (2012) Chemistry and biology of the ubiquitin signal. Angew Chem Int Ed 51:6840-6862 [4] Ciechanover A (2003) The ubiquitin proteolytic system and pathogenesis of human diseases: a novel platform for mechanism-based drug targeting. Biochem Soc Trans 31:474-481

[5] Scheffner M, Nuber U, Huibregtse JM (1995) Protein ubiquitination involving an E1-E2-E3 enzyme ubiquitin thioester cascade. Nature 373:81-83

[6] Thrower JS, Hoffman L, Rechsteiner M et al. (2000) Recognition of the polyubiquitin proteolytic signal. EMBO J 19:94-102

[7] Husnjak K, Dikic I (2012) Ubiquitin-binding proteins: decoders of ubiquitin-mediated cellular functions. Annu Rev Biochem 81:291-322

[8] Hemantha HP, Brik A (2013) Non-enzymatic synthesis of ubiquitin chains: where chemistry makes a difference. Bioorg Med Chem 21:3411-3420

[9] Schneider D, Schneider T, Rösner D et al. (2013) Improving bioorthogonal protein ubiquitylation by click reaction. Bioorg Med Chem 21:3430-3435

[10] Schneider T, Schneider D, Rösner D et al. (2014) Dissecting ubiquitin signaling with linkage-defined and protease resistant ubiquitin chains. Angew Chem Int Ed 53:12925-12929

[11] Rape M (2010) Assembly of k11-linked ubiquitin chains by the anaphase-promoting complex. Subcell Biochem 54:107-115

[12] Rauh NR, Schmidt A, Bormann J et al. (2005) Calcium triggers exit from meiosis II by targeting the APC/C inhibitor XErp1 for degradation. Nature 437:1048-1052

Korrespondenzadresse:

Prof. Dr. Andreas Marx

Prof. Dr. Martin Scheffner

Fachbereiche Chemie und Biologie

Universität Konstanz

Universitätsstraße 10

D-78457 Konstanz

Tel.: 07531-88-5139 (A. Marx),

-5150 (M. Scheffner)

andreas.marx@uni-konstanz.de

martin.scheffner@uni-konstanz.de

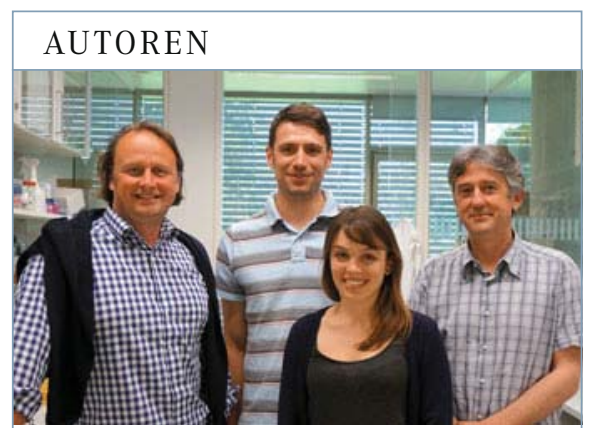

Andreas Marx, Daniel Schneider, Tatjana Schneider und Martin Scheffner (v. I. n. r.)

In einem langjährigen Kooperationsprojekt beschäftigen sich die Gruppen von Prof. Dr. M. Scheffner und Prof. Dr. A. Marx mit den funktionellen Konsequenzen der Ubiquitinierung. Mittels Kombination von molekularbiologischen und chemischen Methoden haben sie neue Konzepte zur spezifischen in vitro-Ubiquitinierung von Proteinen erarbeitet und so neue Möglichkeiten zur Untersuchung des Ubiquitin-Codes entwickelt. 